\title{
Cost -Benefit Analysis of a Single-Unit System with Preventive Maintenance and Weibull Distribution for Failure and Repair Activities
}

\author{
A. KUMAR AND M. SAINI
}

\begin{abstract}
This paper deals with a reliability model developed for a single-unit system which goes for preventive maintenance after a pre-specific time ' $t$ ' up to which no failure occurs. There is a single server who takes some time to arrive at the system for doing repair activities. The unit does not work as new after repair at complete failure and so called the degraded unit. The degraded unit is replaced by new one after its failure with some replacement time. The failure time, preventive maintenance time, replacement time and repair time of the unit are taken as Weibull distributed with common shape parameter and different scale parameters. The switching devices are perfect. The system is observed at suitable regenerative epochs to obtain various measures of system effectiveness of interest to system designers and operation managers.
\end{abstract}

Additional Key Words and Phrases: Single-Unit System, Preventive Maintenance, Degradation, Arrival Time of the Server and Cost-Benefit Analysis.

\section{INTRODUCTION}

In reliability theory, it has been proved that performance and reliability of repairable systems can be improved by the method of redundancy. But there are many systems in which a unit cannot be kept as spare due to its high cost. Therefore, reliability models of single-unit systems with different failure modes have also been probed by the authors including Malik and Bansal [7], Malik [8], Kharoufeh J.P. et al. [5], Malik et al. [9] and Uematsu and Nishida [11] and Pawar and Malik [10] keeping in view of their practical utility and common man's affordability under the following assumptions:

i. The Unit has a constant failure rate.

ii. The unit can work as new after repair and maintenance.

iii. The unit can work forever without conducting preventive maintenance.

iv. Inspection of the failed degraded unit will be conducted to see the feasibility of repair.

v. Server is immediately available as and when required. 
But the instantaneous hazard rates of many systems such as rotating shafts, valves and cams are of non-linear nature due to wear out under mechanical stress and so their failure and repair times follow arbitrary distributions like Weibull distribution. Gupta et al. [3] analyzed a two dissimilar unit cold standby system model by taking Weibull failure and repair distribution. Further, sometimes, unit does not work as new after repair. Since the capability of the unit after repair depends on the repair mechanism adopted and unit may have increased failure rate, if it is repaired by an ordinary server. In such a situation unit becomes degraded. Furthermore, repair of a degraded unit is not always feasible to the system may because of its excessive use and high cost of maintenance. In such cases, the failed degraded unit may be replaced by new one immediately. Kadyan and Promila [4] suggested a reliability model under the concept of degraded unit. Again, the continued operation and ageing of systems gradually reduce their performance and reliability. Therefore, PM of these systems may be conducted after a pre-specific time ' $\mathrm{t}$ ' to slow the deterioration process as well as to restore the systems in a younger age or state. The concept of preventive maintenance has been used by kumar and Malik [6] while analyzing the profit of a computer system with maximum operation time and maximum repair time.

But, in practice, the assumption (v) is not always true due to pre occupations of the repair facility and in such a situation service facility may take some time to arrive at the system. Chander [2] has suggested reliability models of a standby system with arrival time of the server.

The purpose of the present paper is to analyze a single-unit system model by incorporating Weibull distribution for failure, preventive maintenance, replacement and repair times with different scale parameters. The characteristics of Weibull distribution are to represent the three well known phases of failure/repair/replacement/maintenance rates of a unit for different values of shape parameter.

Let the random variable $\mathrm{T}$ denotes the time to failure of an item/device having Weibull distribution, its pdf will be:

$$
f(t)=\theta p t^{p-1} \exp \left(-\theta t^{p}\right) ; t \geq 0 \text { and } \theta, p>0
$$


The reliability/survival function and hazard (failure/repair) rate function for Weibull distribution are given by

$$
R(t)=\exp \left(-\theta t^{p}\right) \text { and } h(t)=\theta p t^{p-1} \quad t \geq 0 \text { and } \theta, p>0
$$

It is important to note that $\mathrm{p}$ and $\theta$ are the shape and scale parameters, respectively. If we put $\mathrm{p}=1$ in $(*)$, Weibull distribution reduces to Exponential distribution and if $\mathrm{p}=2$, it reduces to Rayleigh distribution. The hazard function of this distribution will be constant for $\mathrm{p}=1$, linearly increasing for $\mathrm{p}=2$, non-linearly increasing for $\mathrm{p}$ $>2$ and uniformly decreasing for $\mathrm{p}<1$. Brkic [1] deals with the interval estimation of the parameters of Weibull distribution. The following measures of system effectiveness, useful to system designers and operations managers, are obtained by using regenerative point technique.

1. Steady state transition probabilities and mean sojourn times.

2. Reliability of the system and Mean time to system failure (MTSF).

3. Point-wise and steady state availabilities of the system.

4. Expected busy period of the repairman in time interval $(0, \mathrm{t})$.

5 . Net expected profit earned by the system in time interval $(0, t)$ and in steady state.

\section{SYSTEM DESCRIPTION, NOTATIONS AND STATES OF THE SYSTEM}

The system consists of single-unit which has two modes: Normal (N) mode and total failure (F) mode. There is a single server who takes some time to arrive at the system for doing repair and maintenance activities. The unit becomes degraded after repair. The server replaces the failed degraded unit with some replacement time. Preventive maintenance of the operative unit is carried out after a pre-specific time ' $t$ ' up to which no failure occurs. The failure, repair, preventive maintenance, arrival time of the server, transition rate and replacement time distributions of each unit are taken to be independent having the Weibull density with common shape parameter and different scale parameters as follows:

$$
\begin{aligned}
& h_{i}(t)=\alpha_{i} \eta t^{\eta-1} \exp \left(-\alpha_{i} t^{\eta}\right), \quad g(t)=\theta \eta t^{\eta-1} \exp \left(-\theta t^{\eta}\right), \\
& f_{i}(t)=\beta_{i} \eta t^{\eta-1} \exp \left(-\beta_{i} t^{\eta}\right),
\end{aligned}
$$




$$
\begin{aligned}
& k(t)=\gamma \eta t^{\eta-1} \exp \left(-\gamma t^{\eta}\right), m_{i}(t)=\tau_{i} \eta t^{\eta-1} \exp \left(-\tau_{i} t^{\eta}\right) \text { and } \\
& l(t)=l_{1} \eta t^{\eta-1} \exp \left(-l_{1} t^{\eta}\right), \text { where } \quad t \geq 0 \text { and } \eta, \alpha_{i}, \beta_{i}, \gamma, \theta, \tau_{i}, l_{1}>0
\end{aligned}
$$

respectively for original and degraded unit respectively.

\subsection{Notations}

$\alpha_{i} / \beta_{i}(i=1,2):$ Scale parameter of failure/maintenance time distribution for original and degraded unit.

$\gamma / \theta / l_{1}:$ Scale parameter of arrival time of the server/repair time of the original unit/replacement rate of the degraded unit.

$\tau_{1} / \tau_{2} \quad$ : Scale parameter of transition rate for original and degraded unit.

$\eta \quad$ : Shape parameter of failure/ repair/ arrival time of the server/ preventive maintenance/ transition rate/replacement time distribution for original and degraded unit.

$Q_{i j}(.) / q_{i j}():$. Cdf/pdf of transition time from state $\mathrm{Si}$ to state $\mathrm{Sj}$.

$h_{i}(.) / g_{i}(.) / k(.) / l(.) / m_{i}(.) / f_{i}():$.$\quad Failure/repair/arrival time of$ server/replacement/transition rate/preventive maintenance rates of original and degraded unit having the form.

$h_{i}(t)=\alpha_{i} \eta t^{\eta-1} e^{-\alpha_{i} t^{\eta}}, g(t)=\theta \eta t^{\eta-1} e^{-\theta t^{\eta}}, k(t)=\gamma \eta t^{\eta-1} e^{-\gamma t^{\eta}}, l(t)=l_{1} \eta t^{\eta-1} e^{-l_{1} t^{\eta}}, m_{i}(t)=\tau_{i} \eta t^{\eta-1} e^{-\tau_{i} t^{\eta}}$

and $f_{i}(t)=\beta_{i} \eta t^{\eta-1} e^{-\beta_{i} t^{\eta}} \quad$ where $t \geq 0$ and $\eta, \alpha_{i}, \beta_{i}, \gamma, \theta, \tau_{i}, l_{1}>0$

$p_{i j} \quad:$ Direct/indirect steady state transition probabilities from state $\mathrm{Si}$ to state $\mathrm{Sj}$.

$\psi_{i} \quad:$ Mean sojourn time in state $\mathrm{Si}$

* : Symbol for Laplace Transform.

S : Dummy variable in Laplace Transform. 
2.2. Symbols for the states of the system:

$N_{o} / D_{0} \quad:$ Unit is in original/degraded mode and operative.

Fur / Fwr : Unit is in failure mode and under repair/ waiting for repair.

$\mathrm{Pm} / \mathrm{WPm} / \mathrm{DPm}$ : Unit is under preventive maintenance / waiting for preventive maintenance/ Degraded unit under preventive maintenance.

$D F_{\text {urp }} \quad:$ Failed degraded unit under replacement.

Using these symbols, the system can be in any one of the following states.

$S_{0}=N_{0} \quad S_{1}=W P m \quad S_{2}=P m \quad S_{3}=F w r \quad S_{4}=F u \quad S_{5}=D_{0} \quad S_{6}=$ DFur $S_{7}=D P m$

We observe that the states S0 and S5 are up states and S2, S3, S4, S6 and S7 are failed states. It is also revealed that all the states are regenerative states.

\section{TRANSITION PROBABILITIES AND SOJOURN TIMES}

The transition probability matrix (t.p.m.) of the embedded Markov Chain is

$$
P=\left(\begin{array}{llllllll}
p_{00} & p_{01} & p_{02} & p_{03} & p_{04} & p_{05} & p_{06} & p_{07} \\
p_{10} & p_{11} & p_{12} & p_{13} & p_{14} & p_{15} & p_{16} & p_{17} \\
p_{20} & p_{21} & p_{22} & p_{23} & p_{24} & p_{25} & p_{26} & p_{27} \\
p_{30} & p_{31} & p_{32} & p_{33} & p_{34} & p_{35} & p_{36} & p_{37} \\
p_{40} & p_{41} & p_{42} & p_{43} & p_{44} & p_{45} & p_{46} & p_{47} \\
p_{50} & p_{51} & p_{52} & p_{53} & p_{54} & p_{55} & p_{56} & p_{57} \\
p_{60} & p_{61} & p_{62} & p_{63} & p_{64} & p_{65} & p_{66} & p_{67} \\
p_{70} & p_{71} & p_{72} & p_{73} & p_{74} & p_{75} & p_{76} & p_{77}
\end{array}\right)
$$

With non-zero elements:

$$
\begin{aligned}
& p_{12}=1, p_{20}=1, p_{34}=1, p_{45}=1, p_{60}=1, p_{75}=1, p_{01}=\frac{\tau_{1}}{\tau_{1}+\alpha_{1}}, p_{03}=\frac{\alpha_{1}}{\tau_{1}+\alpha_{1}}, \\
& p_{57}=\frac{\tau_{2}}{\tau_{2}+\alpha_{2}}, p_{56}=\frac{\alpha_{2}}{\tau_{2}+\alpha_{2}}
\end{aligned}
$$

and the other elements of the transition probability matrix will be zero. 
As an illustration, to obtain $p_{45}$, the probability that the system transits from state S4 to state S5 during time interval $(0, \infty)$ we observe as follows:

$p_{45}=\int[$ Probability that repair of the failed unit in state $\mathrm{S} 4$ completes during time $(\mathrm{v}, \mathrm{v}+\mathrm{dv})] \mathrm{dv}$

$$
=\int \theta \eta v^{\eta-1} e^{-\theta v^{\eta}} d v=1
$$

It can be easily verified that $p_{01}+p_{03}=1, p_{56}+p_{57}=1$

The mean sojourn time $\psi_{i}$ in state $S_{i}$ is defined as the expected time taken by the system in state $S_{i}$ before transitioning into any other state. If random variable $U_{i}$ denotes the sojourn time in state $S_{i}$ then $\psi_{i}=\int P\left[U_{i}>t\right] d t$

Therefore, its values for various regenerative states are as follows:

$$
\begin{aligned}
& \psi_{0}=\frac{\Gamma\left(1+\frac{1}{\eta}\right)}{\left(\alpha_{1}+\tau_{1}\right)^{\frac{1}{\eta}}}, \psi_{1}=\psi_{3}=\frac{\Gamma\left(1+\frac{1}{\eta}\right)}{\left(\gamma_{1}\right)^{\frac{1}{\eta}}}, \psi_{2}=\frac{\Gamma\left(1+\frac{1}{\eta}\right)}{\left(\beta_{1}\right)^{\frac{1}{\eta}}}, \psi_{4}=\frac{\Gamma\left(1+\frac{1}{\eta}\right)}{(\theta)^{\frac{1}{\eta}}}, \psi_{5}=\frac{\Gamma\left(1+\frac{1}{\eta}\right)}{\left(\alpha_{2}+\tau_{2}\right)^{\frac{1}{\eta}}}, \psi_{6}=\frac{\Gamma\left(1+\frac{1}{\eta}\right)}{\frac{1}{\frac{1}{\eta}}} \\
& \text { and } \psi_{7}=\frac{\Gamma\left(1+\frac{1}{\eta}\right)}{\left(\beta_{2}\right)^{\frac{1}{\eta}}}
\end{aligned}
$$

As an illustration, to obtain $\psi_{0}$, we observe as follows:

$\psi_{0}=\int\left[\right.$ Probability that the operating unit in state $\mathrm{S}_{0}$ neither fail nor undergoes for PM up to time $\left.\mathrm{t}\right] d t$

$$
=\int e^{-\left(\alpha_{1}+\tau_{1}\right) t^{\eta}} d t=\frac{\Gamma\left(1+\frac{1}{\eta}\right)}{\left(\alpha_{1}+\tau_{1}\right)^{\frac{1}{\eta}}}
$$




\section{ANALYSIS OF RESULTS}

\subsection{Reliability of the system and MTSF}

Let the random variable $T_{i}$ denotes the time to system failure i.e., time taken by the system to reach into any of the failed state, when the system initially starts from regenerative state $S_{i}$, then the reliability of the system is given by $R_{i}(t)=P\left(T_{i}>t\right)$ To determine $R_{i}(t)$, we regard the failed states $S_{i}$ and $S_{3}$ of the system as absorbing. By probabilistic arguments, we have

$$
R_{0}(t)=Z_{0}(t) \text { where } Z_{0}(t)=e^{-\left(\alpha_{1}+\tau_{1}\right) t^{\eta}}
$$

Taking the Laplace Transforms of relations (3) and simplifying for $R_{0}^{*}(s)$, omitting the argument's' for brevity, we get

$$
R_{0}^{*}(s)=Z_{0}^{*}(s)
$$

Taking the Inverse Laplace Transforms of (4), we can get the reliability of the system when it starts from state $S_{0}$. The MTSF when system initially starts from $S_{0}$ is given by,

$$
E\left(T_{0}\right)=\int_{0}^{\infty} R_{0}(t) d t=\lim _{s \rightarrow o} R_{0}^{*}(s)=\psi_{0}
$$

\subsection{Availability analysis}

Let $A_{i}(t)$ be the probability that the system is operative at epoch $\mathrm{t}$ when initially, it starts from regenerative state $S_{i}$. By simple probabilistic arguments, we have the following recursive relations in $A_{i}(t)$ : 


$$
\begin{aligned}
& A_{0}(t)=Z_{0}(t)+q_{03}(t) \Subset A_{3}(t)+q_{01}(t) \Subset A_{1}(t) \\
& A_{1}(t)=q_{12}(t) \Subset A_{2}(t) \\
& A_{2}(t)=q_{20}(t) @ A_{0}(t) \\
& A_{3}(t)=q_{34}(t) \Subset A_{4}(t) \\
& A_{4}(t)=q_{45}(t) \Subset A_{5}(t) \\
& A_{5}(t)=Z_{5}(t)+q_{56}(t) @ A_{6}(t)+q_{57}(t) @ A_{7}(t) \\
& A_{6}(t)=q_{60}(t) \Subset A_{0}(t) \\
& A_{7}(t)=q_{75}(t) \Subset A_{5}(t) \\
& \text { where } Z_{0}(t)=e^{-\left(\alpha_{1}+\tau_{1}\right) t^{\eta}} \text { and } Z_{5}(t)=e^{-\left(\alpha_{2}+\tau_{2}\right) t^{\eta}}
\end{aligned}
$$

Taking the Laplace Transforms of relations (6) and simplifying for $A_{0}^{*}(s)$, omitting the argument's' for brevity, we get

$$
A_{0}^{*}(s)=\frac{Z_{0}^{*}\left[1-q_{57}^{*} q_{75}^{*}\right]+Z_{5}^{*}\left[q_{03}^{*} q_{34}^{*} q_{45}^{*}\right]}{\left[1-q_{57}^{*} q_{75}^{*}\right]-\left[1-q_{57}^{*} q_{75}^{*}\right] q_{20}^{*} q_{12}^{*} q_{01}^{*}-q_{03}^{*} q_{34}^{*} q_{45}^{*} q_{60}^{*} q_{56}^{*}}
$$

Taking the Inverse Laplace Transform of (7), we can get availability of the system for known values of the parameters.

In the long run, the steady state probability that the system will be operative, is given by,

$$
A_{0}=\lim _{t \rightarrow \infty} A_{0}(t)=\lim _{s \rightarrow 0} s A_{0}^{*}(s)=\frac{\psi_{0}\left[1-p_{57} p_{75}\right]+\psi_{5}\left[p_{03} p_{34} p_{45}\right]}{\left[\psi_{0}+p_{01} \psi_{1}+p_{01} \psi_{2}+p_{03}\left\{\psi_{3}+\psi_{4}\right\}\right]\left[1-p_{57}\right]+\left[\psi_{5}+p_{57} \psi_{7}+p_{56} \psi_{6}\right] p_{03}}
$$

The expected up time of the system during time interval $(0, \mathrm{t})$ is

$$
\mu_{u p}(t)=\int_{0}^{t} A_{0}(u) d u \text { so that } \mu_{u p}^{*}(s)=\frac{A_{0}^{*}(s)}{s}
$$

\subsection{Busy period analysis}

Let $B_{i}(t)$ be the probability that the server will be busy at epoch $t$ when initially, its operation starts from regenerative state $S_{i}$. By simple probabilistic arguments, we have the following recursive relations in $B_{i}(t)$ : 


$$
\begin{aligned}
& B_{0}(t)=q_{03}(t) \Subset B_{3}(t)+q_{01}(t) \Subset B_{1}(t) \\
& B_{1}(t)=q_{12}(t) @ B_{2}(t) \\
& B_{2}(t)=Z_{2}(t)+q_{20}(t) \oplus B_{0}(t) \\
& B_{3}(t)=q_{34}(t) \oplus B_{4}(t) \\
& B_{4}(t)=Z_{4}(t)+q_{45}(t) \oplus B_{5}(t) \\
& B_{5}(t)=q_{56}(t) \Subset B_{6}(t)+q_{57}(t) @ B_{7}(t) \\
& B_{6}(t)=Z_{6}(t)+q_{60}(t) \oplus B_{0}(t) \\
& B_{7}(t)=Z_{7}(t)+q_{75}(t) \oplus B_{5}(t) \\
& \text { where } Z_{2}(t)=e^{-\beta_{1} t^{\eta}}, Z_{4}(t)=e^{-\theta t^{\eta}}, Z_{6}(t)=e^{-l_{1} t^{\eta}} \text { and } Z_{7}(t)=e^{-\beta_{2} t^{\eta}}
\end{aligned}
$$

Taking the Laplace Transforms of relations (10) and simplifying for $B_{0}^{*}(s)$, omitting the argument's' for brevity, we get

$$
B_{0}^{*}(s)=\frac{Z_{2}^{*} q_{01}^{*} q_{12}^{*}\left[1-q_{57}^{*} q_{75}^{*}\right]+q_{03}^{*} q_{34}^{*}\left\{Z_{4}^{*}\left[1-q_{57}^{*} q_{75}^{*}\right]+q_{45}^{*}\left[Z_{6}^{*} q_{56}^{*}+Z_{7}^{*} q_{57}^{*}\right]\right\}}{\left[1-q_{57}^{*} q_{75}^{*}\right]-\left[1-q_{57}^{*} q_{75}^{*}\right] q_{20}^{*} q_{12}^{*} q_{01}^{*}-q_{03}^{*} q_{34}^{*} q_{45}^{*} q_{60}^{*} q_{56}^{*}}
$$

Taking the Inverse Laplace Transform of (11) we can get the probability that the server will be busy at a particular epoch for known values of the parameters.

In the long run, the steady state probability that the server will be busy in the repair of a failed unit, is given by,

$$
B_{0}=\lim _{t \rightarrow \infty} B_{0}(t)=\lim _{s \rightarrow 0} s B_{0}^{*}(s)=\frac{\psi_{0} p_{01} p_{12}\left[1-p_{57} p_{75}\right]+p_{03} p_{34}\left\{\psi_{4}\left[1-p_{57} p_{75}\right]+p_{45}\left[\psi_{6} p_{56}+p_{57} \psi_{7}\right]\right\}}{\left[\psi_{0}+p_{01} \psi_{1}+p_{01} \psi_{2}+p_{03}\left\{\psi_{3}+\psi_{4}\right\}\right]\left[1-p_{57}\right]+\left[\psi_{5}+p_{57} \psi_{7}+p_{56} \psi_{6}\right] p_{03}}
$$

The expected busy period of the server during time interval $(0, t)$ is

$$
\mu_{b}(t)=\int_{0}^{t} B_{0}(u) d u \text { so that } \mu_{b}^{*}(s)=\frac{B_{0}^{*}(s)}{s}
$$




\section{COST BENEFIT ANALYSIS}

The net expected profit incurred by the system during time interval $(0, t)$ is given by

$$
C(t)=K_{0} \mu_{u p}(t)-K_{1} \mu_{b}(t)
$$

where $K_{0}$ is the revenue per unit up time by the system and $K_{0}$ is the amount paid to the server per unit time when the system is under repair. Here, we assume that the repair cost of original and degraded unit are same in par unit of time. The net expected profit incurred by the system per unit of time in steady state is given by

$$
C=K_{0} A_{0}-K_{1} B_{0}
$$

where $A_{0}$ and $B_{0}$ has been defined already in previous sections.

\section{CASE STUDIES WITH DISCUSSIONS}

i. When shape parameter $\eta=0.5$ then failure/preventive maintenance/arrival time of the server/replacement/transition rate/ repair time distributions reduce to:

$$
\begin{aligned}
& h_{i}(t)=\frac{\alpha_{i}}{2 \sqrt{t}} e^{-\alpha_{i} \sqrt{t}}, g(t)=\frac{\theta}{2 \sqrt{t}} e^{-\theta \sqrt{t}}, k(t)=\frac{\gamma}{2 \sqrt{t}} e^{-\gamma \sqrt{t}}, l(t)=\frac{l_{1}}{2 \sqrt{t}} e^{-l_{1} \sqrt{t}}, m_{i}(t)=\frac{\tau_{i}}{2 \sqrt{t}} e^{-\tau_{i} \sqrt{t}} \\
& \text { and } f_{i}(t)=\frac{\beta_{i}}{2 \sqrt{t}} e^{-\beta_{i} \sqrt{t}} ; \text { where } t \geq 0 \text { and } \eta, \alpha_{i}, \beta_{i}, \gamma, \theta, \tau_{i}, l_{1}>0
\end{aligned}
$$

So that the results given by (16) will be as follows:

$$
\psi_{0}=\frac{2}{\left(\alpha_{1}+\tau_{1}\right)^{2}}, \psi_{1}=\psi_{3}=\frac{2}{\gamma_{1}^{2}}, \psi_{2}=\frac{2}{\beta_{1}^{2}}, \psi_{4}=\frac{2}{\theta^{2}}, \psi_{5}=\frac{2}{\left(\alpha_{2}+\tau_{2}\right)^{2}}, \psi_{6}=\frac{2}{l_{1}^{2}} \text { and } \psi_{7}=\frac{2}{\beta_{2}^{2}}
$$

ii. When shape parameter $\eta=1.0$ then failure/preventive maintenance/arrival time of the server/replacement/transition rate/ repair time distributions reduce to exponentials, then 
$h_{i}(t)=\alpha_{i} e^{-\alpha_{i} t}, g(t)=\theta e^{-\theta t}, k(t)=\gamma e^{-\gamma t}, l(t)=l_{1} e^{-l_{1} t}, m_{i}(t)=\tau_{i} e^{-\tau_{i} t}$ and $f_{i}(t)=\beta_{i} e^{-\beta_{i} t}$;

where $t \geq 0$ and $\eta, \alpha_{i}, \beta_{i}, \gamma, \theta, \tau_{i} l_{1}>0$

So that the results given by (18) will be as follows:

$\psi_{0}=\frac{1}{\left(\alpha_{1}+\tau_{1}\right)}, \psi_{1}=\psi_{3}=\frac{1}{\gamma_{1}}, \psi_{2}=\frac{1}{\beta_{1}}, \psi_{4}=\frac{1}{\theta}, \psi_{5}=\frac{1}{\left(\alpha_{2}+\tau_{2}\right)}, \psi_{6}=\frac{1}{l_{1}}$ and $\psi_{7}=\frac{1}{\beta_{2}}$

iii. When shape parameter $\eta=2.0$ then failure/preventive maintenance/arrival time of the server/replacement/transition rate/ repair time distributions reduce to Rayleigh having the pdf-

$h_{i}(t)=2 \alpha_{i} t e^{-\alpha_{i} t^{2}}, g(t)=2 \theta t e^{-\theta t^{2}}, k(t)=2 \gamma t e^{-\gamma t^{2}}, l(t)=2 l_{1} t e^{-l_{1} t^{2}}, m_{i}(t)=2 \tau_{i} t e^{-\tau_{i} t^{2}}$ and $f_{i}(t)=2 \beta_{i} t e^{-\beta_{i} t^{2}}$ where $t \geq 0$ and $\eta, \alpha_{i}, \beta_{i}, \gamma, \theta, \tau_{i} l_{1}>0$

So that the results given by (20) will be as follows:

$\psi_{0}=\frac{1}{2} \sqrt{\frac{\pi}{\left(\alpha_{1}+\tau_{1}\right)}}, \psi_{1}=\psi_{3}=\frac{1}{2} \sqrt{\frac{\pi}{\gamma_{1}}}, \psi_{2}=\frac{1}{2} \sqrt{\frac{\pi}{\beta_{1}}}, \psi_{4}=\frac{1}{2} \sqrt{\frac{\pi}{\theta}}, \psi_{5}=\frac{1}{2} \sqrt{\frac{\pi}{\left(\alpha_{2}+\tau_{2}\right)}}, \psi_{6}=\frac{1}{2} \sqrt{\frac{\pi}{l_{1}}}$ and $\psi_{7}=\frac{1}{2} \sqrt{\frac{\pi}{\beta_{2}}}$

By using the MATLAB software, we have obtained the values of reliability characteristics (MTSF, availability and net expected steady state profit) of the system for above three cases for different values of the parameters in Tables 1, 3 and 5. In table 1, MTSF of above three cases are shown with respect to $\alpha_{1}$ for two different values of $\tau_{1}(0.5,0.6)$. The values of other parameters are kept fixed as $\left(\alpha_{2=0.8}, \tau_{2}=1.2, l_{1}=1.4, \beta_{1}=1.7, \beta_{2}=1.6, \theta=0.5\right.$ and $\left.\gamma=5\right)$.

Similarly, in table 3 the values for availability are shown for the same values of all the parameters as in the case of MTSF. 
Table 1 Values of MTSF for different values of $\tau_{1}$ and $\eta$ with respect to $\alpha_{1}$

\begin{tabular}{|c|c|c|c|c|c|c|}
\hline \multirow[t]{2}{*}{$\alpha_{1}$} & \multicolumn{2}{|c|}{$\eta=0.5$} & \multicolumn{2}{|c|}{$\eta=1.0$} & \multicolumn{2}{|c|}{$\eta=2.0$} \\
\hline & $\tau_{1}=0.12$ & $\tau_{1}=0.18$ & $\tau_{1}=0.12$ & $\tau_{1}=0.18$ & $\tau_{1}=0.12$ & $\tau_{1}=0.18$ \\
\hline 0.10 & 41.3223 & 25.5102 & 4.5455 & 3.5714 & 1.8894 & 1.6748 \\
\hline 0.11 & 37.8072 & 23.7812 & 4.3478 & 3.4483 & 1.8479 & 1.6457 \\
\hline 0.12 & 34.7222 & 22.2222 & 4.1667 & 3.3333 & 1.8090 & 1.6180 \\
\hline 0.13 & 32.0000 & 20.8117 & 4.0000 & 3.2258 & 1.7725 & 1.5917 \\
\hline 0.14 & 29.5858 & 19.5313 & 3.8462 & 3.1250 & 1.7380 & 1.5666 \\
\hline 0.15 & 27.4348 & 18.3655 & 3.7037 & 3.0303 & 1.7055 & 1.5427 \\
\hline 0.16 & 25.5102 & 17.3010 & 3.5714 & 2.9412 & 1.6748 & 1.5199 \\
\hline 0.17 & 23.7812 & 16.3265 & 3.4483 & 2.8571 & 1.6457 & 1.4980 \\
\hline 0.18 & 22.2222 & 15.4321 & 3.3333 & 2.7778 & 1.6180 & 1.4770 \\
\hline 0.19 & 20.8117 & 14.6092 & 3.2258 & 2.7027 & 1.5917 & 1.4569 \\
\hline 0.20 & 19.5313 & 13.8504 & 3.1250 & 2.6316 & 1.5666 & 1.4376 \\
\hline
\end{tabular}

Similarly, in table 5 the values of profit function are shown for the same values of all the parameters as in the case of MTSF and availability. The values of $K_{0}$ and $K_{1}$ for profit function are assumed as $\left(K_{0}=1200, K_{1}=600\right)$.

From table 1, we observed that the MTSF decreases more rapidly as the shape parameter $\eta$ increases. Also with the increase of the value $\alpha_{1}$, MTSF decreases uniformly irrespective of the values of $\eta$ and $\tau_{1}$. Moreover, as the value of $\tau_{1}$ increases from 0.12 to 0.18 , we observe that the MTSF decreases.

Table 2 Values of MTSF for different values of $\tau_{1}$ and $\eta$ with respect to $\alpha_{1}$

\begin{tabular}{|c|c|c|c|c|c|c|}
\hline \multirow[t]{2}{*}{$\alpha_{1}$} & \multicolumn{2}{|c|}{$\eta=0.9$} & \multicolumn{2}{|c|}{$\eta=1.0$} & \multicolumn{2}{|c|}{$\eta=1.1$} \\
\hline & $\tau_{1}=0.12$ & $\tau_{1}=0.18$ & $\tau_{1}=0.12$ & $\tau_{1}=0.18$ & $\tau_{1}=0.12$ & $\tau_{1}=0.18$ \\
\hline 0.10 & 5.6589 & 4.3287 & 4.5455 & 3.5714 & 3.8220 & 3.0695 \\
\hline 0.11 & 5.3862 & 4.1632 & 4.3478 & 3.4483 & 3.6706 & 2.9732 \\
\hline 0.12 & 5.1374 & 4.0093 & 4.1667 & 3.3333 & 3.5313 & 2.8829 \\
\hline 0.13 & 4.9096 & 3.8659 & 4.0000 & 3.2258 & 3.4026 & 2.7982 \\
\hline 0.14 & 4.7003 & 3.7319 & 3.8462 & 3.1250 & 3.2834 & 2.7186 \\
\hline 0.15 & 4.5072 & 3.6064 & 3.7037 & 3.0303 & 3.1727 & 2.6436 \\
\hline 0.16 & 4.3287 & 3.4888 & 3.5714 & 2.9412 & 3.0695 & 2.5729 \\
\hline 0.17 & 4.1632 & 3.3782 & 3.4483 & 2.8571 & 2.9732 & 2.5059 \\
\hline 0.18 & 4.0093 & 3.2741 & 3.3333 & 2.7778 & 2.8829 & 2.4426 \\
\hline 0.19 & 3.8659 & 3.1759 & 3.2258 & 2.7027 & 2.7982 & 2.3825 \\
\hline 0.20 & 3.7319 & 3.0832 & 3.1250 & 2.6316 & 2.7186 & 2.3254 \\
\hline
\end{tabular}


Table 3 Values of Availability for different values of $\tau_{1}$ and $\eta$ with respect to $\alpha_{1}$

\begin{tabular}{|c|c|c|c|c|c|c|}
\hline \multirow[t]{2}{*}{$\alpha_{1}$} & \multicolumn{2}{|c|}{$\eta=0.5$} & \multicolumn{2}{|c|}{$\eta=1.0$} & \multicolumn{2}{|c|}{$\eta=2.0$} \\
\hline & $\tau_{1}=0.12$ & $\tau_{1}=0.18$ & $\tau_{1}=0.12$ & $\tau_{1}=0.18$ & $\tau_{1}=0.12$ & $\tau_{1}=0.18$ \\
\hline 0.10 & 0.8917 & 0.8617 & 0.7010 & 0.6810 & 0.5469 & 0.5373 \\
\hline 0.11 & 0.8784 & 0.8472 & 0.6870 & 0.6679 & 0.5401 & 0.5313 \\
\hline 0.12 & 0.8649 & 0.8325 & 0.6738 & 0.6556 & 0.5339 & 0.5257 \\
\hline 0.13 & 0.8511 & 0.8177 & 0.6613 & 0.6440 & 0.5283 & 0.5205 \\
\hline 0.14 & 0.8371 & 0.8029 & 0.6496 & 0.6330 & 0.5230 & 0.5158 \\
\hline 0.15 & 0.8230 & 0.7881 & 0.6385 & 0.6227 & 0.5182 & 0.5113 \\
\hline 0.16 & 0.8088 & 0.7734 & 0.6280 & 0.6128 & 0.5138 & 0.5072 \\
\hline 0.17 & 0.7945 & 0.7587 & 0.6180 & 0.6035 & 0.5096 & 0.5033 \\
\hline 0.18 & 0.7802 & 0.7441 & 0.6086 & 0.5946 & 0.5057 & 0.4997 \\
\hline 0.19 & 0.7659 & 0.7297 & 0.5996 & 0.5862 & 0.5021 & 0.4963 \\
\hline 0.20 & 0.7517 & 0.7154 & 0.5910 & 0.5781 & 0.4987 & 0.4931 \\
\hline
\end{tabular}

Further, from tables 3 and 5, we observe that availability and profit function of the system model are also follows the same trend as MTSF. Also with the increase of the value $\alpha_{1}$, availability and profit decreases uniformly irrespective of the values of $\eta$ and $\tau_{1}$. Moreover, as the value of $\tau_{1}$ increases from 0.12 to 0.18 , we observe that the availability and profit decreases. Here one thing is important that if we increase shape parameter $\eta$ and decrease $\tau_{1}$ then system is more profitable in comparison to the situation when $\eta$ and $\tau_{1}$ both increases.

Table 4 Values of Availability for different values of $\tau_{1}$ and $\eta$ with respect to $\alpha_{1}$

\begin{tabular}{|c|c|c|c|c|c|c|}
\hline \multirow[t]{2}{*}{$\alpha_{1}$} & \multicolumn{2}{|c|}{$\eta=0.9$} & \multicolumn{2}{|c|}{$\eta=1.0$} & \multicolumn{2}{|c|}{$\eta=1.1$} \\
\hline & $\tau_{1}=0.12$ & $\tau_{1}=0.18$ & $\tau_{1}=0.12$ & $\tau_{1}=0.18$ & $\tau_{1}=0.12$ & $\tau_{1}=0.18$ \\
\hline 0.10 & 0.7314 & 0.7089 & 0.7010 & 0.6810 & 0.6747 & 0.6567 \\
\hline 0.11 & 0.7165 & 0.6950 & 0.6870 & 0.6679 & 0.6616 & 0.6446 \\
\hline 0.12 & 0.7024 & 0.6817 & 0.6738 & 0.6556 & 0.6493 & 0.6332 \\
\hline 0.13 & 0.6891 & 0.6691 & 0.6613 & 0.6440 & 0.6378 & 0.6226 \\
\hline 0.14 & 0.6763 & 0.6572 & 0.6496 & 0.6330 & 0.6270 & 0.6125 \\
\hline 0.15 & 0.6642 & 0.6458 & 0.6385 & 0.6227 & 0.6168 & 0.6031 \\
\hline 0.16 & 0.6527 & 0.6349 & 0.6280 & 0.6128 & 0.6073 & 0.5942 \\
\hline 0.17 & 0.6417 & 0.6245 & 0.6180 & 0.6035 & 0.5983 & 0.5857 \\
\hline 0.18 & 0.6312 & 0.6147 & 0.6086 & 0.5946 & 0.5897 & 0.5778 \\
\hline 0.19 & 0.6212 & 0.6052 & 0.5996 & 0.5862 & 0.5817 & 0.5702 \\
\hline 0.20 & 0.6116 & 0.5962 & 0.5910 & 0.5781 & 0.5740 & 0.5630 \\
\hline
\end{tabular}


Table 5 Values of Profit for different values of $\tau_{1}$ and $\eta$ with respect to $\alpha_{1}$

\begin{tabular}{crrrrrrrr}
\hline \multirow{2}{*}{$\alpha_{1}$} & \multicolumn{2}{c}{$\eta=0.5$} & & \multicolumn{2}{c}{$\eta=1.0$} & & \multicolumn{2}{c}{$\eta=2.0$} \\
\cline { 2 - 3 } \cline { 7 - 8 } & $\tau_{1}=0.12$ & $\tau_{1}=0.18$ & & $\tau_{1}=0.12$ & $\tau_{1}=0.18$ & & $\tau_{1}=0.12$ & $\tau_{1}=0.18$ \\
\hline 0.10 & 990.7216 & 924.6307 & & 604.2070 & 538.0979 & & 310.6588 & 257.4500 \\
0.11 & 966.5537 & 898.0719 & & 581.4166 & 517.9208 & & 304.5149 & 253.6795 \\
0.12 & 941.8709 & 871.3215 & & 559.9886 & 498.9173 & & 298.8430 & 250.1478 \\
0.13 & 916.7710 & 844.4643 & & 539.8044 & 480.9880 & & 293.5810 & 246.8277 \\
0.14 & 891.3473 & 817.5793 & & 520.7588 & 464.0443 & & 288.6777 & 243.6963 \\
0.15 & 865.6881 & 790.7393 & & 502.7581 & 448.0072 & & 284.0909 & 240.7339 \\
0.16 & 839.8768 & 764.0108 & & 485.7186 & 432.8057 & & 279.7853 & 237.9241 \\
0.17 & 813.9909 & 737.4542 & & 469.5653 & 418.3762 & & 275.7307 & 235.2525 \\
0.18 & 788.1023 & 711.1243 & & 454.2308 & 404.6614 & & 271.9018 & 232.7067 \\
0.19 & 762.2775 & 685.0696 & & 439.6544 & 391.6094 & & 268.2765 & 230.2758 \\
0.20 & 736.5768 & 659.3336 & & 425.7813 & 379.1733 & & 264.8360 & 227.9503 \\
\hline
\end{tabular}

Table 6 Values of Profit for different values of $\tau_{1}$ and $\eta$ with respect to $\alpha_{1}$

\begin{tabular}{|c|c|c|c|c|c|c|}
\hline \multirow[t]{2}{*}{$\alpha_{1}$} & \multicolumn{2}{|c|}{$\eta=0.9$} & \multicolumn{2}{|c|}{$\eta=1.0$} & \multicolumn{2}{|c|}{$\eta=1.1$} \\
\hline & $\tau_{1}=0.12$ & $\tau_{1}=0.18$ & $\tau_{1}=0.12$ & $\tau_{1}=0.18$ & $\tau_{1}=0.12$ & $\tau_{1}=0.18$ \\
\hline 0.10 & 664.9017 & 596.5038 & 604.2070 & 538.0979 & 552.0869 & 488.2269 \\
\hline 0.11 & 640.0125 & 573.9096 & 581.4166 & 517.9208 & 531.5169 & 470.4229 \\
\hline 0.12 & 616.4046 & 552.4823 & 559.9886 & 498.9173 & 512.3054 & 453.7411 \\
\hline 0.13 & 593.9899 & 532.1394 & 539.8044 & 480.9880 & 494.3167 & 438.0751 \\
\hline 0.14 & 572.6876 & 512.8055 & 520.7588 & 464.0443 & 477.4331 & 423.3319 \\
\hline 0.15 & 552.4232 & 494.4116 & 502.7581 & 448.0072 & 461.5518 & 409.4297 \\
\hline 0.16 & 533.1282 & 476.8946 & 485.7186 & 432.8057 & 446.5829 & 396.2962 \\
\hline 0.17 & 514.7393 & 460.1965 & 469.5653 & 418.3762 & 432.4470 & 383.8674 \\
\hline 0.18 & 497.1985 & 444.2644 & 454.2308 & 404.6614 & 419.0740 & 372.0862 \\
\hline 0.19 & 480.4520 & 429.0493 & 439.6544 & 391.6094 & 406.4014 & 360.9018 \\
\hline 0.20 & 464.4504 & 414.5062 & 425.7813 & 379.1733 & 394.3738 & 350.2685 \\
\hline
\end{tabular}

\section{CONCLUSIONS}

For a more concrete study of the single-unit system under preventive maintenance and Weibull distributed failure and repair activities, we obtain numerical results for MTSF, availability and profit function in tables 2,4, and 6 respectively, for different values of $\tau_{1}(0.12,0.18)$ and $\eta(0.9,1.0,1.1)$ against the failure parameter $\alpha_{1}$, while the others parameters are kept fixed as: $\alpha_{2=0.8}, \tau_{2}=1.2, l_{1}=1.4$, 
$\beta_{1}=1.7, \beta_{2}=1.6, \theta=0.5, \gamma=5, K_{0}=1200, K_{1}=600$. From Tables 2,4 and 6

respectively, we observe that MTSF, availability and profit function decreases with

the increase in $\eta, \alpha_{1}$ and $\tau_{1}$.

\section{REFERENCES}

1. Brkic, D.M.: Interval estimation of the parameters $\beta$ and $\eta$ of the two parameter Weibull distribution. Microelectron Reliab. 30(1):39-42,1990.

2. Chander, S.: Reliability models with priority for operation and repair with arrival time of server, Pure and Applied Mathematika Sciences, Vol. LXI, No. 1-2, pp. $9-22,2005$.

3. Gupta, R., Kumar, P. and Gupta, A.: Cost-Benefit analysis of a two dissimilar unit cold standby system with Weibull failure and repair laws. Int. J. Syst. Assur. Eng. Manag. 4(4):327-334, 2013.

4. Kadyan, M.S., Promila, Reliability and Cost-Benefit Analysis of a Single Unit System with Degradation and Inspection at Different Stages of Failure subject to Weather Conditions. International Journal of Computer Applications, Volume 55- No.6, 33-38,2012.

5. Kharoufeh J.P., Solo C.J. and Ulukus M.Y.: Semi-Markov models for degradation- based reliability, IIE Transactions, 42, 599-612, 2010.

6. Kumar, A. and Malik, S. C. (2011): Profit Analysis of a Computer System with Priority to Software Replacement over Hardware Repair Subject to Maximum Operation and Repair Times, International Journal of Engineering Science \& Technology, Vol.3, No. 10, pp. 7452- 7468.

7. Malik,S.C. and Bansal, R.K.( 2005) : Profit analysis of single-unit reliability models with repair at different failure modes. Proc. International Conference on Reliability and Safety Engineering, IIT Kharagpur (India), pp. 577-588.

8. Malik,S.C. (2008) : Reliability modeling and profit analysis of a single-unit system with inspection by a server who appears and disappears randomly. Journal of Pure and Applied Mathematika Sciences, Vol.LXVII(1-2), pp. 135-146.

9. Malik, S.C., Chand, P. and Singh, J.: Stochastic analysis of an operating system with two types of inspection subject to degradation, Applied Probability and Statistics, 3(2), 227-241,2008.

10. Pawar, D. and Malik,S.C. (2010): Reliability and Economic Measures of a System with Inspection for Online Repair and No Repair Activity in Abnormal Weather. Bulletin of Pure and Applied Sciences, Vol. 29 E(2),pp.355-368.

11. Uematsu, K. and Nishida, T.: One-unit system with a failure rate depending upon the degree of repair, Mathematica Japonica, 32/1, 139-147, 1987.

Ashish Kumar

Department of Mathematics,

Manipal University,

Jaipur-303007, Rajasthan (India)

Email: ashishbarak2020@gmail.com

\section{Monika Saini}

Department of Mathematics, Manipal University, Jaipur-303007, Rajasthan (India) 